\title{
Perfil de competencias y batería de técnicas psicodiagnósticas para la selección del cargo dependiente gastronómico. Estudio de caso
}

\section{Profile of competences and battery of psychodiagnostic techniques \\ for the selection of the gastronomic dependent position. A case study}

Dra. Arianne Medina Macías ${ }^{1}$

Lic. Gabriela Cabrera Lorenzo ${ }^{2}$

Lic. Yaily Fabia González Borrego ${ }^{3}$

\begin{abstract}
RESUMEN
El modelo de gestión del capital humano por competencias se emplea en un gran número de organizaciones cubanas. Su base radica en el perfil de competencias del cargo; por ello, es esta una de las demandas más frecuentes que se le realizan a la Facultad de Psicología en sus prácticas preprofesionales de Psicología Laboral y Organizacional. Es a esto que tributan los resultados de la presente investigación. El objetivo es diseñar un perfil de competencias del cargo "dependiente gastronómico" (DA) de una cooperativa de servicios gastronómicos, a partir del cual se elabora una propuesta de batería de técnicas para la selección de personal en el cargo en cuestión. Se emplea la metodología cualitativa con un diseño fenomenológico. Se utilizaron como técnicas: análisis documental, guía general de la organización, cuestionario, diario de actividades, entrevistas semiestructuradas y el método minidelphi. Se obtuvieron tres resultados principales: en primer lugar, las condiciones laborales beneficiosas y nocivas para el desempeño en el cargo; en segundo lugar, el perfil de competencias del cargo conformado por cinco competencias claves: trabajo en equipo, dominio técnico de la gastronomía, comportamiento ético, comunicación interpersonal y orientación al servicio y en tercer lugar, la batería de técnicas psicodiagnósticas integrada por: entrevista semiestructurada, Locus de Control de Rotter y dos técnicas de simulación-

Palabras clave: competencias laborales, perfil de competencias, condiciones laborales, batería de técnicas psicodiagnósticas, dependiente gastronómico.
\end{abstract}

\footnotetext{
${ }^{1}$ Universidad de La Habana, Profesora Titular de la Facultad de Psicología. La Habana, Cuba. Correo electrónico: arianne@psico.uh.cu ORCID: https://orcid.org/0000-0002-6473-1190

${ }^{2}$ Universidad de la Habana. Adiestrada de la Facultad de Psicología. La Habana, Cuba. Correo electrónico: gabriela.cabrera@psico.uh.cu ORCID: https://orcid.org/0000-0002-3813-7550

${ }^{3}$ Universidad de La Habana. Adiestrada de la Facultad de Psicología. La Habana, Cuba. Correo electrónico: yaily.gonzalez@psico.uh.cu ORCID: https://orcid.org/0000-0002-3707-0550.

DOI: $10.15517 /$ WL.V16l1.46859
}

Recepción:13/10/2020 Aceptación: 5/4/2021 


\begin{abstract}
The human capital management model by competencies is used in a large number of Cuban organizations. Its basis lies in the skill profile of the position; For this reason, this is one of the most frequent demands that are made to the Faculty of Psychology in its pre-professional practices of Labor and Organizational Psychology. It is to this that the results of the present investigation pay tribute. The objective is to design a competency profile for the "gastronomic clerk" (DA) position of a gastronomic services cooperative, from which a proposal for a battery of techniques is prepared for the selection of personnel in the position in question. The qualitative methodology is used with a phenomenological design. The following techniques were used: documentary analysis, general organization guide, questionnaire, activity diary, semi-structured interviews and the minidelphi method. Three main results were obtained: first, beneficial and harmful working conditions for the performance of the position; second, the competency profile of the position made up of five key competencies: teamwork, technical mastery of gastronomy, ethical behavior, interpersonal communication and service orientation. Thirdly, the battery of psychodiagnostic techniques made up of: semi-structured interview, Rotter's Locus of Control and two simulation techniques.

Key Words: job skills, skills profile, working conditions, battery of psychodiagnostic techniques, gastronomic dependent.
\end{abstract}

\title{
Introducción
}

El abordaje de los problemas relativos al vínculo entre el individuo y la organización es de vital importancia para lograr la productividad del trabajo y el desarrollo individual, grupal, organizacional, e, inclusive, el social; esto en un contexto donde las personas son las que verdaderamente añaden valor a la organización. El estudio de tales procesos y fenómenos organizacionales se ha llevado a cabo desde múltiples aproximaciones teóricas, las cuales han contribuido a desarrollar y fortalecer el objeto de estudio de la Psicología Laboral y Organizacional, y las funciones que el profesional de la Psicología puede desempeñar en el ámbito organizacional.

Entre las funciones más importantes del psicólogo organizacional, se visualiza la gestión de capital humano $(\mathrm{GCH})$, labor que debe asumir el desarrollo de las personas en el trabajo como objetivo primordial, alineado al logro de las metas organizacionales. Entre los procesos llevados a cabo para la GCH se encuentran: la selección del personal idóneo para determinado cargo; el diseño de puestos de trabajo y la organización del trabajo; la evaluación del desempeño; la compensación; la capacitación, formación y el desarrollo de carreras; etcétera. En el contexto internacional la GCH por competencias se ha difundido en gran medida por la posibilidad de alinear el desempeño individual con el organizacional 
(alineación estratégica) $^{4,5,6}$; así como por su enfoque de procesos, al determinarse las competencias que permitirán no solo obtener un desempeño superior en el cargo, sino también la gestión de interrelaciones, logro de sinergias y un trabajo cooperativo. ${ }^{7}$

Considerando la diversidad de conceptos de competencias laborales que existen en la literatura, en el presente trabajo se adopta el referido en la Norma Cubana NC 3000:2007:

Conjunto sinérgico de conocimientos, habilidades, experiencias, sentimientos, actitudes, motivaciones, características personales y valores, basado en la idoneidad demostrada, asociado a un desempeño superior del trabajador y de la organización y que se encuentran en correspondencia con las exigencias técnicas, productivas y de servicios. Estas competencias deben ser observables, medibles y, a su vez, contribuir al logro de los objetivos de la organización. ${ }^{8}$

En este sentido, se reconoce el carácter sinérgico de los elementos de la competencia, su necesaria contextualización y la posibilidad de que esta sea visible y fácil de medir en el desempeño.

Las competencias laborales requeridas para el desempeño de un cargo quedan recogidas en un perfil de competencias, el cual se considera: un "documento que describe las competencias requeridas para un cargo y expresa la relación de los objetivos estratégicos y metas con las capacidades que debe desarrollar el personal de la organización” (NC 3000:2007, 19). Según Cárdenas, Pérez, González y Marrero dicho perfil depende, en primera instancia, de la función que desarrolle el puesto de trabajo en cuestión, así como de la cultura de cada empresa en particular. ${ }^{9}$. Por lo que cada organización adecúa los perfiles de competencia según los objetivos que persiga.

Es necesario aclarar que, dicho documento, no abarca necesariamente todas las funciones y tareas del cargo, pues al definir un perfil se marcarán sólo las competencias más

\footnotetext{
${ }^{4}$ Cuesta, Armando. "Modelo Integrado de gestión humana y del conocimiento: una tecnología de aplicación". Revista venezolana de Gerencia, 17, no. 57 (2012): 86-98

${ }^{5}$ Medina, Arianne y Vitier, Amanda. "Socializando buenas prácticas sobre diseño de perfiles de competencias". Alternativas Cubanas en Psicología (ACUPSI) 4, no. 10 (2016): 57-82.

${ }^{6}$ Medina, Arianne y Vitier, Amanda. "¿La gestión de competencias influye en la efectividad organizacional? Apuntes para la reflexión desde la práctica en empresas cubanas". Revista Wimb lu, 11, no.2 (2016a): 59-85.

7 Soltura, Ariel y Cuesta, Armando. "Diseño estratégico de perfiles de cargos por competencias. Una contribución al alineamiento del desempeño individual con el desempeño organizacional". Ingeniería Industrial XXIX, no. 1 (2008): 52-56. http://www.redalyc.org/articulo.oa?id=360433565003.

${ }^{8}$ Oficina Nacional de Normalización. Norma Cubana 3000:2007. Sistema de Gestión Integrada de Capital Humano-Vocabulario. Vedado. La Habana. Cuba. 2007: 10

${ }^{9}$ Cárdenas, Elia Teresa., Pérez, Olga Liset, González, Aliesky y Marrero, Fernando. "Manual de trabajo para diseñar los perfiles de cargos por competencias en las Universidades". Uniandes EPISTEME. Revista digital de Ciencia, Tecnología e Innovación, 7, no. 2 (2020): 207-219. ISSN 1390-9150.
} 
importantes (claves) para obtener buenos resultados en ese puesto en particular y el grado requerido para cada una de ellas. ${ }^{10}$

El cargo seleccionado para el presente estudio fue "dependiente gastronómico" de una cooperativa de servicios gastronómicos. Este posee como misión fundamental: ofrecer un servicio gastronómico de calidad al cliente, contribuyendo a su satisfacción. En cuanto a la entidad empresarial, es una organización que pertenece al sector de Cooperativas No Agropecuarias (CNA), coloquialmente conocido como "negocio privado o por cuenta propia"; ubicada en el municipio Centro Habana. Según sus objetivos y funciones esenciales, es una organización de servicios. Posee una administración colectiva, constituida por varios socios.

El objetivo que se persigue en el presente estudio es diseñar un perfil de competencias del cargo “dependiente gastronómico" (DA) de una cooperativa no agropecuaria, a partir del cual se elabora una propuesta de batería de técnicas para la selección de personal en el cargo en cuestión.

El trabajo ${ }^{11}$ realizado por las autoras de la investigación de referencia, reporta beneficios a la empresa en cuestión, pues facilita su actualización en modelos de desarrollo de capital humano, lo cual resulta importante para su eficiencia, permite conocer la relación entre las exigencias del cargo y las condiciones laborales existentes. A la misma vez, posibilita identificar efectos funcionales y disfuncionales asociados al desempeño en el cargo de dicha organización. En suma, se favorece el perfeccionamiento de los procesos de selección y reclutamiento de personal con un método científico basado en el perfil de competencias.

Así mismo, el empleo del perfil elaborado reportará otros beneficios a mayor escala, tal y como plantea Labrada:

La posibilidad de focalizar de manera individual y grupal aquellos comportamientos del cargo en cuestión que producen los resultados requeridos por la organización; alinear las herramientas de gestión de las personas e integrar estas herramientas de gestión a todo el sistema de la entidad; tributando a los sistemas de gestión integrada. $^{12}$

\footnotetext{
${ }^{10}$ Alles, Martha. "Perfil del puesto por competencias. Sepa cómo construirlo y evitar bajos desempeños posteriores". (2020). http://www.tblgroup.com.

${ }^{11}$ El artículo deriva del trabajo de curso realizado por un grupo de cinco estudiantes de 3er año de Psicología de la Universidad de La Habana en la asignatura Psicología Laboral en las Organizaciones, bajo la tutoría de la Dra. Arianne Medina, profesora titular de la asignatura.

${ }^{12}$ Labrada, Edel. "Perfil de competencias del personal de servicios gastronómicos en la UEB servicios a trabajadores de la empresa de construcción y montaje de Las Tunas". Tesis en opción al título académico de
} 
Los resultados obtenidos resultaron de gran utilidad para los ocupantes del cargo, ya que permiten que la organización los comprenda como sujetos activos y capaces de llevar a la empresa al éxito a partir de sus competencias. Todo esto tributa al desarrollo de los individuos y la organización.

\section{Diseño Metodológico}

\section{Selección de la muestra}

Se utilizaron dos tipos de muestra: de expertos (ocupantes del cargo con mayor experiencia) y de informantes claves (jefe inmediato superior y encargado de la GCH). Ambas se seleccionaron de forma intencional, considerando los siguientes criterios.

Criterios de selección de la muestra de expertos:

- Poseer, cada experto, como mínimo, tres años de experiencia en el cargo.

- Conocimiento vasto del cargo objeto de estudio. (Preferiblemente ocupantes del cargo o personas que han tenido contacto directo y prolongado con el mismo; por ejemplo: ex - ocupantes o jefes inmediatos superiores).

- Gozar de buenos resultados de trabajo (a partir de evaluaciones de desempeño anteriores y criterio del jefe inmediato superior).

Criterios de selección de la muestra de informantes claves:

- Contar, como mínimo, con un año de experiencia en la entidad.

- Ocupar cargos que aportan información relevante para el estudio.

Máster en Dirección. Facultad de Ciencias Económicas Centro de Estudios de Dirección. Universidad de Las Tunas. 2018: 17. 
Wimblu, Rev. Estud. de Psicología UCR, 16(1) 2021 (Enero-Junio): 75-101 /ISSN: 1659-2107

Caracterización de las muestras:

Tabla 1. Caracterización sociodemográfica de la muestra de expertos (ocupantes del cargo).

\begin{tabular}{clcccc}
\hline ID. & Cargo & Sexo & Edad & $\begin{array}{c}\text { Años de experiencia } \\
\text { en la organización }\end{array}$ & $\begin{array}{c}\text { Años de experiencia } \\
\text { en el cargo }\end{array}$ \\
\hline A & $\begin{array}{l}\text { Dependiente Gastronómico } \\
\text { (Restaurante) }\end{array}$ & F & 25 & 1 año & 3 años \\
B & $\begin{array}{l}\text { Dependiente Gastronómico } \\
\text { (Bar) }\end{array}$ & F & 27 & 1 año & 5 años \\
C & $\begin{array}{l}\text { Dependiente Gastronómico } \\
\text { (Bar) }\end{array}$ & M & 29 & 1 año & 9 años \\
D & $\begin{array}{l}\text { Dependiente Gastronómico } \\
\text { (Bar) }\end{array}$ & M & 30 & 2 años & 8 años \\
E & $\begin{array}{l}\text { Dependiente Gastronómico } \\
\text { (Bar) }\end{array}$ & F & 27 & 2 años & 5 años \\
\hline
\end{tabular}

Tabla 2. Caracterización sociodemográfica de la muestra de informantes claves

\begin{tabular}{llcccc}
\hline ID. & Cargo & Sexo & Edad & $\begin{array}{l}\text { Años de experiencia en } \\
\text { la organización }\end{array}$ & $\begin{array}{l}\text { Años de experiencia en } \\
\text { el cargo }\end{array}$ \\
\hline A & RR.HH & M & 53 & 4 años & 4 años \\
B & $\begin{array}{l}\text { Control y } \\
\text { Fiscalización }\end{array}$ & M & 75 & 4 años & 3 años \\
Capitán de & M & 28 & 2 años & 1 año \\
salón
\end{tabular}

Metodología

Se recurre a una metodología cualitativa caracterizada, según Hernández, Fernández y Baptista $^{13}$ por los siguientes elementos:

- La inmersión inicial en el campo implica sensibilizarse con el ambiente en el cual se lleva a cabo el estudio; así como identificar informantes que aporten datos y guíen al investigador por el lugar, adentrarse y compenetrarse con la situación de investigación, además de verificar la factibilidad del estudio.

- La muestra, la recolección y el análisis son fases que se realizan, prácticamente, de manera simultánea.

- Utiliza técnicas para recolectar datos, tales como: la observación no estructurada, entrevistas abiertas, revisión de documentos, discusión en grupo, evaluación de experiencias personales, registro de historias de vida, e interacción e introspección con grupos o comunidades.

${ }^{13}$ Hernández, R., Fernández, C. y Baptista, P. Metodología de la Investigación. México: Mc. Grau Hill Education. 2014. 
El tipo de diseño de la investigación es fenomenológico, pues permite explorar, describir y comprender las experiencias de las personas con respecto a un fenómeno. Así mismo, tiene como propósito descubrir los elementos en común de tales vivencias, haciendo uso de herramientas de recolección de información como la observación, entrevistas personales o grupos de enfoque con preguntas abiertas, semiestructuradas y estructuradas, artefactos, documentos variados, grabaciones en audio y video e, incluso, instrumentos estandarizados. 14

Técnicas y métodos

Análisis documental: se consultaron documentos relevantes de la organización: planeación estratégica, historia de la organización, organigrama, manual de protección e higiene del trabajo.

Guía General de la Organización: se utilizó la guía del manual de la asignatura Psicología Laboral en las Organizaciones ${ }^{15}$ para caracterizar la empresa, teniendo en cuenta sus particularidades: misión, visión y objetivos estratégicos, aspectos físicos, etc.

Cuestionario para caracterizar la GRH: se utilizó la guía del manual de la asignatura Psicología Laboral en las Organizaciones ${ }^{12}$ para caracterizar los procesos de GRH: reclutamiento, selección de personal y seguridad y salud en el trabajo.

Diario de actividades: Esta técnica permitió obtener, en un registro lo más exhaustivo posible, las tareas desarrolladas por los dependientes gastronómicos en un día típico de trabajo, a partir del horario laboral (Ver Anexo 1).

Entrevista semiestructurada de condiciones laborales: Se basa en un modelo de condiciones enriquecido de la propuesta de los autores Straub y Hacker ${ }^{16}$. A través de esta técnica se describen las condiciones laborales internas y externas del cargo objeto de estudio, así como su efecto en los resultados del trabajo (Ver Anexo 2).

14 Hernández, R., Fernández, C., \& Baptista, P. Metodología de la Investigación. México: Mc. Grau Hill Education. 2014: 493.

${ }^{15}$ Medina, Arianne; Martínez, Marta; Vitier, Amanda; Blanco, Yaima y Hernández, Midalys. "Manual de Trabajo Extraclase de Psicología Laboral en las Organizaciones para el diseño de perfiles de cargos por competencias". Facultad de Psicología. Universidad de La Habana. 2019.

${ }^{16}$ Medina, Arianne y Vitier, Amanda. "El perfil de competencias: comparación de procedimientos para su diseño en el ámbito académico y empresarial cubano". Integración Académica en Psicología, 5, no. 15 (2016b): 107-124. ISSN 2007-5588. 110 
Wimblu, Rev. Estud. de Psicología UCR, 16(1) 2021 (Enero-Junio): 75-101 /ISSN: 1659-2107

Entrevista de incidentes críticos: Su objetivo radica en la indagación sobre experiencias de éxito y de fracaso en el desempeño del cargo de los expertos. Con su utilización se exploran los siguientes aspectos: situaciones laborales críticas, grados de recurrencia competencias puestas en juego por el experto para afrontar dificultades y los criterios de desempeño superior (Ver Anexo 3).

Método minidelphi. Se utilizó una adaptación del Método Delphi propuesto por Cuesta (adaptado por Medina et al., 2019) ${ }^{17}$. El procedimiento consiste en obtener opiniones y, preferiblemente, un consenso, de un grupo de expertos con relación a las competencias claves para el desempeño superior del cargo. La adaptación utilizada consiste en entregar una lista preliminar de las competencias del cargo objeto de estudio a los expertos, la cual deriva del análisis triangulado de la información recopilada en las técnicas anteriormente aplicadas.

\section{Análisis de los resultados}

Los resultados obtenidos se presentan en la secuencia de los objetivos específicos de la investigación. Se siguió un enfoque que hace énfasis en el desempeño competente, el cual siguiendo a Medina y Vitier ${ }^{13}$ rescata la dimensión del poder hacer, elemento que, con frecuencia, está ausente en las propuestas que se realizan sobre la gestión de competencias laborales. De modo que los resultados obtenidos contribuyen a una concepción del desempeño superior del trabajador en el cargo contextualizado, debido a que el desempeño de éxito no solo depende de las competencias propias del trabajador, sino también de las condiciones laborales óptimas que se requieren para alcanzar este estándar de desempeño, de las cuales pueden derivar, también, efectos disfuncionales.

Análisis de las condiciones laborales.

El análisis de las condiciones laborales fue realizado a partir de: la información integrada del grupo de expertos con relación a la entrevista semiestructurada de condiciones laborales (Ver Anexo 2), las observaciones, la guía general de la organización, el análisis de los documentos del cargo, las normas de protección e higiene del trabajo y el diario de actividades.

\footnotetext{
17 Medina, Arianne; Martínez, Marta; Vitier, Amanda; Blanco, Yaima y Hernández, Midalys. "Manual de Trabajo Extraclase de Psicología Laboral en las Organizaciones para el diseño de perfiles de cargos por competencias". Facultad de Psicología. Universidad de La Habana. 2019.
} 
Wimblu, Rev. Estud. de Psicología UCR, 16(1) 2021 (Enero-Junio): 75-101 /ISSN: 1659-2107

De forma general se diagnosticó que, en su mayoría, las condiciones laborales generan efectos funcionales para el desempeño de la actividad laboral. Sin embargo, el horario laboral extenso y el régimen de trabajo y descanso (trabajo en días alternos) generan efectos disfuncionales a mediano o largo plazo, como la fatiga y el estrés; por lo que es necesaria su modificación, con vistas a prevenir la aparición de estados que afecten la salud de los dependientes y su desempeño laboral.

A continuación, se exponen los principales resultados del análisis cualitativo de las condiciones laborales y los efectos que producen. En el grupo de las condiciones relevantes para el desempeño superior que resultan beneficiosas según su efecto, se encuentran las siguientes:

\section{Condiciones sociopsicológicas:}

El estilo de dirección es autocrático; mientras que el de relaciones interpersonales es de colaboración. La comunicación es vertical descendente/ascendente, horizontal, transversal. La selección de estas condiciones sociopsicológicas como relevantes para el desempeño superior, coincide con lo encontrado por Cáceres en la sistematización sobre el diseño de perfiles de 20 cargos en organizaciones laborales de La Habana. ${ }^{18}$

Estas condiciones generan efectos funcionales en los trabajadores de la organización. Los motiva a cumplir con los requerimientos necesarios para el desempeño de su actividad laboral y los requerimientos de los directivos contribuyen a potenciar el desarrollo de los dependientes. Por su parte, el establecimiento de relaciones de colaboración y vías de comunicación eficientes facilitan la obtención de mejores resultados laborales en un clima social más ameno y satisfactorio, lo cual repercute en el bienestar de los trabajadores, en su motivación y en un servicio de mayor calidad. Llama la atención que se encontró también en Cáceres un efecto beneficioso en la mayoría de las condiciones laborales; incluso, en el caso de la dirección autocrática, se encontró que este tipo de estilo puede asociarse a efectos funcionales, lo que demuestra el potencial carácter bivalente y mediado psíquicamente de las condiciones laborales. ${ }^{15}$

\footnotetext{
18 Cáceres, Ana Beatriz. "Impacto de las condiciones laborales en los resultados del trabajo en cargos de organizaciones laborales de La Habana receptoras de prácticas de Psicología Laboral”. Tesis en opción al título de Licenciado en Psicología. Facultad de Psicología. Universidad de La Habana. 2020.
} 


\section{Condiciones organizativas:}

La organización de dos turnos de trabajo (se trabaja en días alternos); hay remuneración (sistema de pago por rendimiento con fecha fija); estrategias de estimulación (fomento de la moral). Estas condiciones, según la valoración de los expertos, generan motivaciones extrínsecas hacia el trabajo. Sin embargo, el reconocimiento moral al buen trabajador, no solo desde los directivos, sino también dentro del equipo de trabajo, se traduce en una mayor motivación intrínseca. Las motivaciones intrínsecas a la actividad laboral se relacionan, en lo fundamental, con necesidades de logro, autorrealización, autodeterminación y desarrollo personal.

Las condiciones ambientales (relaciones espaciales del local, luz e iluminación, ruido, microclima, son de confort):

Los dependientes reportan sentirse cómodos en su puesto de trabajo. Estas condiciones permiten brindar un servicio de mayor calidad. La evaluación afectiva y/o cognitiva de las experiencias de trabajo generan efectos funcionales, por ejemplo, la satisfacción laboral. Lo mismo sucede con los medios de trabajo, como condición propia del puesto de trabajo.

Condiciones específicas de la actividad:

La misión del cargo (brindar un servicio gastronómico de calidad y lograr la satisfacción de los clientes de la instalación); el ritmo de trabajo acelerado e impuesto por la gran afluencia de clientes, en especial en los horarios pico (tarde-noche); las normas de trabajo (de calidad y de disciplina laboral); el trabajo en equipo, con necesidades de cooperación y colaboración; y un grado de libertad medio-bajo en la toma de decisiones (los dependientes deben recurrir siempre a su capitán de salón) caracterizan la actividad laboral misma. Como efectos resultantes de estas condiciones, los expertos reportan la satisfacción, la motivación intrínseca y extrínseca hacia el trabajo y la fatiga. Como se espera en la relación dialéctica condiciones-efectos, estos últimos pueden tener un carácter bivalente. Para este puesto, los dependientes consideran que, si bien el ritmo de trabajo les puede provocar fatiga, también significa mayor remuneración y estimulación. Además, el desempeño adecuado de su actividad laboral constituye una recompensa importante para ellos. A su vez, el trabajo en equipo y la cooperación elevan el nivel de motivación y generan un efecto funcional de satisfacción laboral. Son evidentes los mecanismos de mediación psíquica de las condiciones laborales y de compensación que permiten reevaluar los efectos disfuncionales generados. 
Premisas psicofísicas estables del rendimiento:

Los dependientes gastronómicos deben tener: buen estado de los órganos de los sentidos (se utilizan, en especial: la vista, el tacto y el oído, no pueden tener ninguna discapacidad de estos); buen estado de salud general, una constitución física de promedio fuerte; conocimientos técnicos sobre cómo se sirven y retiran los platos, la adecuación de cada cubierto, sobre alimentos, cocina y cantina; maridaje, cultura general sobre el país, dominio de idiomas (fundamentalmente inglés) y sobre inventario. Según reportan los expertos, estas condiciones generan efectos funcionales relacionados con la satisfacción laboral y el desarrollo de la personalidad (aprenden en el contacto con clientes y en el propio desempeño). Estos son elementos muy importantes, porque se relacionan con motivaciones intrínsecas a la actividad laboral. Las posibilidades de desarrollo y de autorrealización inciden no solo en la motivación, sino también en su satisfacción laboral.

Otras de estas premisas estables del rendimiento son: habilidades y destrezas físicas (agilidad en el manejo de bandejas, platería, etcétera, coordinación viso espacial y óculomanual); actitudes hacia el trabajo (respeto, tolerancia); rasgos del carácter (cordial, cortés, amable, flexible); motivación extrínseca y extrínseca; estado de entrenamiento y experiencia (meses de entrenamiento y preparación previa). El poseer ciertos conocimientos, habilidades y destrezas posibilita que el trabajador experimente sentimientos, emociones y estados de ánimo positivos, relacionados con autoeficacia y bienestar. De igual forma, repercute en su satisfacción laboral, generando afectividad positiva por parte del trabajador, en tanto se siente vinculado con su entorno de trabajo.

Algunos testimonios de lo que refieren los expertos al respecto son los siguientes: de rasgos del carácter: "carácter flexible, que se acomode a todo tipo de situación, para que no se afecte el trabajo"; de actitudes hacia el trabajo: "para ser un buen dependiente debe gustarte lo que haces, el trato con el cliente y la gastronomía en general"; de habilidades o destrezas: "rápido, preciso, expresarte bien, ser sociable (por ejemplo, saber entretener a un niño) y saber idiomas".

De las condiciones laborales presentadas, los expertos valoraron como relevantes y de efecto beneficioso para el desempeño laboral óptimo más del 50\%. Lo anterior demuestra la complejidad del cargo estudiado y la importancia de velar por un buen diseño de las condiciones presentadas con anterioridad. Resulta importante señalar que, en su mayoría, 
generan efectos funcionales en los resultados de la actividad laboral, incluyendo los estados en el trabajador que ocupa el cargo, lo que coincide con los resultados de Cáceres. ${ }^{19}$

Las condiciones relacionadas con la organización del trabajo: horario laboral extenso (Bar: 10:00am-12:00pm y Restaurante: 8:00am-12:00pm); ritmo de trabajo (acelerado en horarios pico, impuesto por la afluencia de clientes); régimen de trabajo y descanso (se trabaja en días alternos), postura física (de pie, cargando peso y desplazándose); tipo de gasto (físico y emocional); riesgos predominantes (físicos, ergonómicos y psicosociales) son consideradas relevantes para el desempeño superior del cargo, y con efectos nocivos para el trabajador y los resultados de trabajo. Vale destacar el carácter determinante que tienen estas condiciones sobre la seguridad y salud en el trabajador, debido a su exposición a los riesgos laborales, en especial los ergonómicos y psicosociales. ${ }^{20}$. Como efectos disfuncionales se reconoce la fatiga, coincidiendo con uno de los estados disfuncionales más frecuentes de la investigación de Cáceres. ${ }^{16}$. Los empleados aluden que, “después de varias horas de trabajo ya uno no está en condiciones (tanto físicas como psicológicas) de prestar un servicio de calidad, aunque se tenga la mayor motivación, pues el cansancio, la fatiga y el estrés te impiden continuar como al inicio”. Esto muestra la disminución del rendimiento dada la intensidad de la actividad que realizan y su prolongación en el tiempo. Se experimenta una sensación general de cansancio y agotamiento, disminuyendo su capacidad de concentración en el trabajo y aumentando su irritabilidad, síntomas que tipifican el estado de fatiga. Esto obstaculiza el desempeño superior en el cargo, por lo que se deberían reevaluar las causas que la provocan, para prevenirla. De no garantizarse estas condiciones en su estado óptimo, el desempeño laboral requerirá de un sobresfuerzo del trabajador y un mayor desgaste que conduce a una disminución del rendimiento, lo cual provocará efectos disfuncionales a mediano o a largo plazo, por ejemplo, la fatiga y el estrés, entre otras. ${ }^{21}$

\footnotetext{
${ }^{19}$ Cáceres, Ana Beatriz. "Impacto de las condiciones laborales en los resultados del trabajo en cargos de organizaciones laborales de La Habana receptoras de prácticas de Psicología Laboral”. Tesis en opción al título de Licenciado en Psicología. Facultad de Psicología. Universidad de La Habana. 2020.

${ }^{20}$ Medina Macías, Arianne y Avila Vidal, Adalberto. “¿Gestión de riesgos psicosociales? Un S.O.S. en organizaciones cubanas". Psicología: Teoría e Práctica, 21, no.2 (2019):191-210.

${ }^{21}$ Avila, Adalberto, y Medina, Arianne. "La Psicología y su relación con la efectividad de las organizaciones laborales". Tabloide de Universidad para Todos (2015). ISBN 978-959-270-333-9.
} 
Diagrama de exigencias laborales

A partir de la información recogida, se identifican las exigencias de la actividad laboral del cargo de dependiente gastronómico, siguiendo la clasificación de Quaas y Raum, (citado en Medina, Martínez, Vitier y Blanco, 2019). Esto es fruto de la aplicación de varias técnicas como: los documentos relacionados con el desempeño del cargo, el modelo de condiciones laborales, el diario de actividades, y las observaciones realizadas.

Tabla 3. Exigencias laborales del dependiente gastronómico según la clasificación de Quass y Raum

\section{Exigencias de tipo 1: Brindar un servicio gastronómico de calidad y la satisfacción del} cliente

\section{Exigencias de tipo 2:}

A. Montar adecuadamente todas las mesas del establecimiento.

B. Contar y hacer inventario de los insumos y los materiales con los que se cuenta para la jornada laboral.

C. Recibir y despedir cordialmente a todos los clientes.

D. Explicarle la carta a los clientes y la forma en que se preparan los alimentos y bebidas que en ella aparecen.

E. Mostrar amabilidad y saber interactuar con todos los clientes que visitan el establecimiento.

F. Recoger los pedidos de los clientes.

G. Llevar y traer los pedidos de los clientes rápidamente.

H. Estar atento a las necesidades de todos los clientes de su área.

I. Saber explicar a los clientes, principalmente extranjeros, la vida y tradiciones culturales del país.

J. Cumplir las normativas y reglamentos que se encuentran establecidos en la entidad.

\section{Exigencias de tipo3:}

1. Adaptabilidad a condiciones de trabajo que suponen un gran esfuerzo físico.

2. Veracidad en las informaciones que se brinde a los clientes, tanto en temas relacionados con la propia empresa, como otros aspectos de interés para los visitantes.

3. Discreción en cuanto a información relacionada con los clientes y con la organización a la que pertenecen.

4. Capacidad para cumplir con calidad y en los términos establecidos la prestación de servicios y la preparación del local para la jornada de trabajo.

5. Buen gusto y creatividad.

6. Capacidad de coordinación.

7. Conocimientos de gastronomía.

8. Rapidez y agilidad en el servicio.

9. Capacidad de concentración y atención.

10. Honestidad e integridad.

11. Capacidad de planificación y organización.

12. Habilidades de comunicación y buen vocabulario.

13. Buenos modales.

14. Carácter flexible y capacidad para gestionar situaciones conflictivas.

15. Conocimientos de cocina y coctelería.

16. Buena escucha, visión y memoria.

17. Conocimientos sobre la cultura y la cotidianidad del país.

18. Dominio básico de idiomas. 
Wimblu, Rev. Estud. de Psicología UCR, 16(1) 2021 (Enero-Junio): 75-101 /ISSN: 1659-2107

Tabla 4. Diagrama de exigencias laborales (correspondencia entre las exigencias tipos 1, 2 y 3 )

\begin{tabular}{|c|c|c|}
\hline Exigencias de tipo 3 & Exigencias de tipo 2: & Exigencia de tipo 1: \\
\hline $4 ; 5 ; 6 ; 7 ; 8$ & $\mathrm{~A}$ & \multirow{10}{*}{$\begin{array}{l}\text { Brindar un servicio } \\
\text { gastronómico de calidad y } \\
\text { lograr la satisfacción de } \\
\text { los clientes de la } \\
\text { instalación. }\end{array}$} \\
\hline $4 ; 8 ; 9 ; 10 ; 11 ; 16$ & $\mathrm{~B}$ & \\
\hline $1 ; 4 ; 12 ; 13 ; 16 ; 18$ & $\mathrm{C}$ & \\
\hline $2 ; 4 ; 7 ; 9 ; 12 ; 13 ; 15 ; 16 ; 18$ & $\mathrm{D}$ & \\
\hline $1 ; 2 ; 3 ; 9 ; 10 ; 12 ; 13 ; 14 ; 16 ; 18$ & $\mathrm{E}$ & \\
\hline $4 ; 8 ; 9 ; 12 ; 14 ; 16 ; 18$ & $\mathrm{~F}$ & \\
\hline $1 ; 4 ; 6 ; 7 ; 8 ; 11 ; 14 ; 16$ & $\mathrm{G}$ & \\
\hline $4 ; 8 ; 9 ; 11 ; 12 ; 16$ & $\mathrm{H}$ & \\
\hline $2 ; 3 ; 10 ; 12 ; 17 ; 18$ & I & \\
\hline $1 ; 3 ; 4 ; 16$ & $\mathrm{~J}$ & \\
\hline
\end{tabular}

Análisis del Método minidelphi

El listado preliminar de competencias presentado a los expertos resultó de la integración de las técnicas previamente aplicadas. Se resumen en este acápite las fases y rondas de aplicación del método minidelphi de consulta a expertos.

La lista de competencias ponderadas por los expertos (a partir del menor valor de la media) quedó de la siguiente manera:

1. Trabajo en equipo $(\mathrm{M}=1.4)$

2. Dominio técnico de la gastronomía $(M=1.6)$

3. Comportamiento ético $(\mathrm{M}=3.6)$

4. Comunicación interpersonal $(\mathrm{M}=4)$

5. Orientación al servicio $(\mathrm{M}=4.4)$

Los niveles de desarrollo requeridos para el desempeño superior en el cargo quedan establecidos como se ilustra en la tabla 5.

Tabla 5. Perfil de competencias requerido para el desempeño superior en el cargo

\begin{tabular}{llllc}
\hline \multicolumn{1}{c}{ COMPETENCIAS } & \multicolumn{2}{c}{ NIVELES DE DESARROLLO REQUERIDOS } \\
& PARTIDA & DESARROLLO & AVANZADO & EXPERTO \\
\hline Trabajo en equipo & & & & $\mathrm{X}$ \\
$\begin{array}{l}\text { Dominio técnico de la } \\
\text { gastronomía } \\
\text { Comportamiento ético }\end{array}$ & & & $\mathrm{X}$ \\
& & & \\
Comunicación interpersonal & & & $\mathrm{X}$ \\
Orientación al servicio & & $\mathrm{X}$ & \\
\hline
\end{tabular}

Leyenda: X Nivel de desarrollo óptimo (requerido) para el cargo. 
A continuación, se expondrá de forma detallada el perfil de competencias del cargo objeto de estudio con el diseño de las competencias claves (definición conceptual, dimensiones competenciales e indicadores comportamentales), resultado del análisis integrado de las técnicas aplicadas y validado por el criterio de expertos; método que goza de amplia aceptación en la investigación científica cubana. ${ }^{22}$

En este perfil quedan implícitos aspectos que Soltura y Cuesta plantean como necesarios para su estructura y contenido: denominación del cargo, misión del cargo, requisitos mínimos para ocupar el cargo, competencias de desempeño superior y sus dimensiones, responsabilidades y condiciones de trabajo. ${ }^{23}$

Tabla 6. Perfil de competencias del cargo

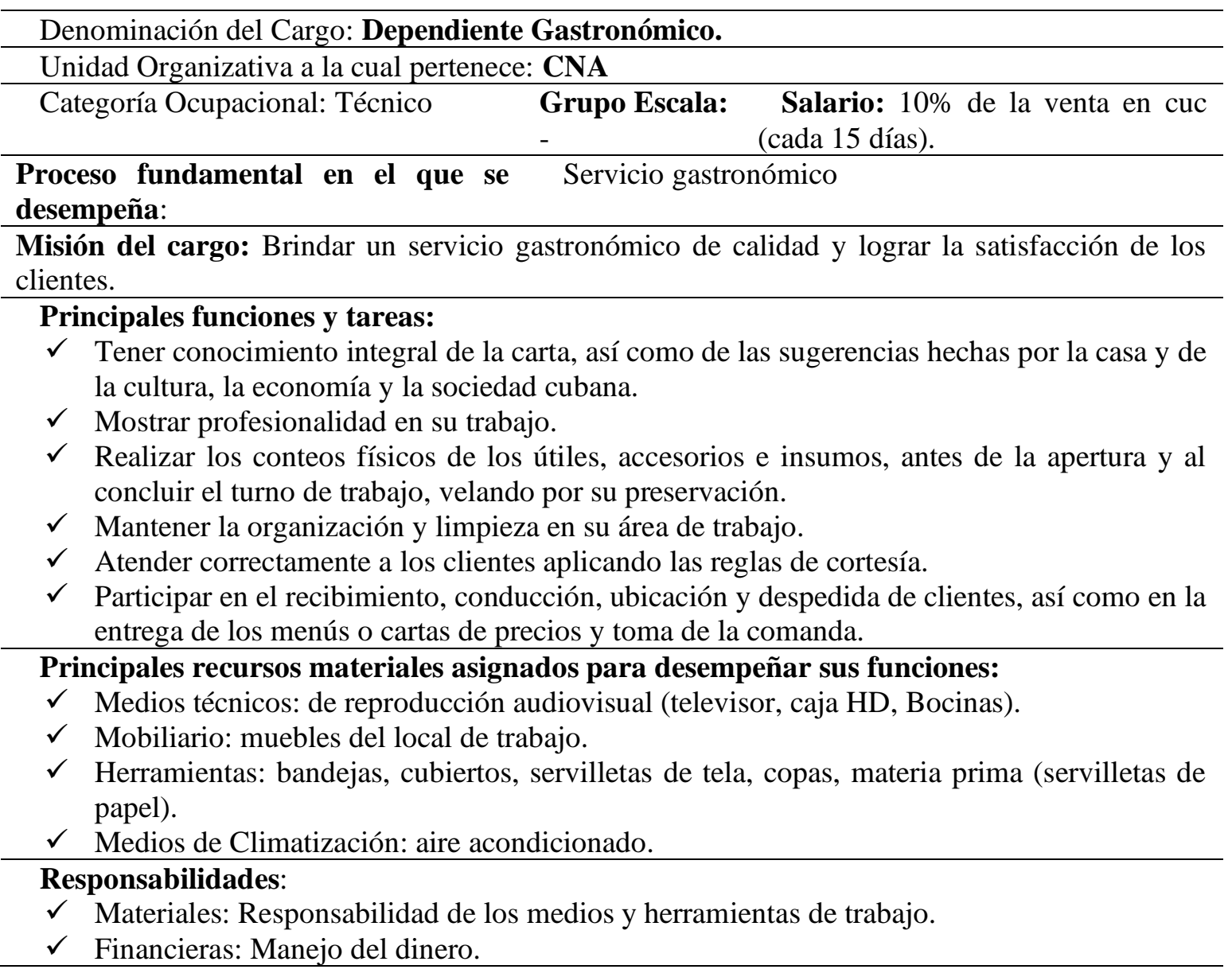

${ }^{22}$ Díaz, Yohan, Cruz, Miguel, Pérez, Milagro de la Caridad y Ortiz, Tania. "El método criterio de expertos en las investigaciones educacionales: visión desde una muestra de tesis doctorales". Revista Cubana de Educación Superior, 39, no.1, e18 (2019). ISSN 0257-4314.

${ }^{23}$ Soltura, Ariel y Cuesta, Armando. "Diseño estratégico de perfiles de cargos por competencias. Una contribución al alineamiento del desempeño individual con el desempeño organizacional". Ingeniería Industrial XXIX, no. 1 (2008): 52-56. http://www.redalyc.org/articulo.oa?id=360433565003. 
Morales: Manejo honesto de la propina, ya que se divide equitativamente entre todos los dependientes, ofrecer una buena imagen de la entidad.

\section{Condiciones de Trabajo \\ Horario de Trabajo:}

Bar: 10:00am-12:00pm

Restaurante: 8:00am-12:00pm

Ambiente de Trabajo: confort.

$\checkmark$ Locales muy bien amueblados.

$\checkmark$ Espacio suficiente para recibir todos los clientes y que los dependientes se desplacen sin problemas.

$\checkmark$ Sistema de ventilación adecuado.

$\checkmark \quad$ Iluminación adecuada para prestar sus servicios.

$\checkmark$ Esfuerzo físico y mental necesario: actividad física elevada.

$\checkmark$ Principales riesgos y enfermedades profesionales a las que está expuesto: Dolores en la columna, las piernas y pies, molestias en el tendón de la mano.

$\checkmark$ Medios de protección necesarios: zapatos ergonómicos, medios de protección contra incendios, cámaras de vigilancia y arcos de seguridad.

\begin{tabular}{ll}
\hline \multicolumn{2}{l}{ DIMENSIONES DE LOS REQUISITOS PERSONALES: } \\
\hline CARGO NIVEL ACADEMICO & Técnico Medio \\
\hline ESPECIALIDADES PREFERENTES & Técnico medio en gastronomía \\
\hline EXPERIENCIA EN EL TEMA & Al menos de 1 año. \\
\hline
\end{tabular}

\begin{tabular}{|c|c|}
\hline \multicolumn{2}{|r|}{ Diseño de las competencias } \\
\hline \multicolumn{2}{|r|}{ 1. Trabajo en equipo } \\
\hline \multicolumn{2}{|c|}{ Nivel de desarrollo óptimo de la competencia para el cargo: Experto } \\
\hline \multicolumn{2}{|c|}{$\begin{array}{l}\text { Definición de la competencia: } \\
\text { Es la capacidad de trabajar con otros para conseguir metas comunes. Supone colaboración y } \\
\text { coordinación entre los miembros del equipo de gastronómicos de la entidad. }\end{array}$} \\
\hline \multicolumn{2}{|l|}{ COMPETENCIALES } \\
\hline \multirow{2}{*}{\multicolumn{2}{|c|}{$\begin{array}{lll}1.1 \text { Colaboración } & \text { y } & \text { 1.1.1 Intercambia información con sus colegas. } \\
\text { cooperación. } & 1.1 .2 \text { Apoya a sus colegas con sus responsabilidades. } \\
& \text { 1.1.3 Busca la ayuda de otros trabajadores, como cocineros, } \\
& \text { asistentes de limpieza y capitanes de salón. } \\
& \text { 1.1.4 Aporta nuevas ideas atractivas para los clientes para el } \\
& \text { beneficio de la actividad laboral y el equipo de trabajo. }\end{array}$}} \\
\hline & \\
\hline 1.2 Respeto hacia el otro. & $\begin{array}{l}\text { 1.2.1 Respeta los estilos de trabajo de sus colegas. } \\
\text { 1.2.2 Respeta distintos puntos de vista sobre la realización del } \\
\text { trabajo. }\end{array}$ \\
\hline $\begin{array}{l}1.3 \text { Organización } y \\
\text { distribución del espacio } \\
\text { físico. }\end{array}$ & $\begin{array}{l}\text { 3.1 Atiende su área física de trabajo. } \\
\text { 3.2 Apoya cuando otras áreas están sobrecargadas de clientes. }\end{array}$ \\
\hline Nombre de la competencia & 2. Dominio técnico de la gastronomía \\
\hline \multicolumn{2}{|c|}{ Nivel de desarrollo óptimo de la competencia para el cargo: Experto } \\
\hline \multicolumn{2}{|c|}{$\begin{array}{l}\text { Definición de la competencia: } \\
\text { Conocer la correcta manipulación y empleo del equipamiento, utensilios, accesorios e insumos, } \\
\text { así como la ejecución de los diferentes tipos y modalidades del servicio gastronómico, de acuerdo } \\
\text { con las tendencias actuales y las características distintivas de la empresa. }\end{array}$} \\
\hline
\end{tabular}


Wimblu, Rev. Estud. de Psicología UCR, 16(1) 2021 (Enero-Junio): 75-101 /ISSN: 1659-2107

\section{DIMENSIONES COMPETENCIALES}

2.2 Buena memoria.

2.2 Conocimiento integral de la carta, cocina y cantina.

\section{DESCRIPTIVOS COMPORTAMENTALES}

2.1.1 Recuerda información personal sobre los clientes repitentes, tales como nombres y gustos.

2.2.1 Domina todos los componentes de la carta: alimentos, características y su cocción, bebidas disponibles, maridajes.

2.2.2 Brinda información sobre el contenido de la carta a los clientes y está atento a sus dudas e inquietudes.

2.2.3 Realiza sugerencias atractivas a los clientes.

2.2.4 Ejecuta con destreza y profesionalidad el servicio de vinos.

2.2.5 Cumple con las normas higiénicas sanitarias en la manipulación de alimentos y otros insumos.
2.3 Manipulación y empleo correcto del equipamiento, utensilios e insumos.

\subsubsection{Dispone los utensilios según las normas técnicas}

2.3.2 Realiza los conteos físicos de los útiles, accesorios e insumos antes de la apertura y al concluir el turno de trabajo, esto para cuidar su preservación.

2.3.3 Mantiene en buen estado, cuidado y control de equipos, útiles y otros medios de trabajo puestos a su disposición.

2.3.4 Reporta cualquier anomalía, rotura u otros desperfectos que surjan en su turno.

2.3.5 Vela por el uso racional de los recursos y materiales asignados para el desempeño del trabajo, inclusive fuera de su radio de acción.

2.4 Profesionalismo en el trato con clientes y colegas de trabajo.

2.4.2 Recibe, conduce, ubica y despide a los clientes con respeto, cortesía y cordialidad, así como en la entrega de los menús o cartas de precios y toma de la comanda. 2.4.1 Se muestra atento y dispuesto a las instrucciones, orientaciones e indicaciones del Capitán de Salón para la realización de su trabajo.

2.4.3 Actúa conforme a las normas y a los estándares técnicos establecidos en la gastronomía.

2.4.4 Genera credibilidad y confianza en los demás.

\section{Nombre de la competencia 3. Comportamiento ético}

Nivel de desarrollo óptimo de la competencia para el cargo: Experto

Definición de la competencia:

Capacidad para comportarse acorde a las directrices, normas y valores de la empresa y de la gastronomía, dando muestras de un elevado nivel de compromiso, así como de profesionalidad y confiabilidad en el actuar.

\section{DIMENSIONES \\ DESCRIPTIVOS COMPORTAMENTALES \\ COMPETENCIALES}

3.1 Asunción de normas de la 3.1.1 Interioriza y actúa acorde a directrices, normas y valores empresa.

de la empresa y de la gastronomía.

3.1.2 Mantiene la disciplina, la buena asistencia y puntualidad.

3.2 Adecuada apariencia física. 3.2.2 Mantiene un porte y aspecto de acuerdo con las reglas establecidas por la casa.

3.2.2 No consume cigarros, tabacos, ni bebidas alcohólicas dentro de las instalaciones.

3.3 Discreción y honestidad. 3.3.1 Muestra total discreción en el manejo de información sensible, tanto de los clientes como de la entidad. 
3.3.2 Alerta y comparte con las instancias establecidas, eventos en los que identifique manifestaciones de corrupción o ilegalidad.

3.3.3 No extrae de la instalación ningún tipo o cantidad de alimento o bebida, procesada o sin procesar.

\section{Nombre de la competencia \\ 4. Comunicación interpersonal}

Nivel de desarrollo óptimo de la competencia para el cargo: Avanzado

Definición de la competencia:

Ser capaz de mediar con los clientes de la instalación y compañeros de trabajo, facilitando la obtención de resultados satisfactorios de la actividad laboral.

\section{DIMENSIONES DESCRIPTIVOS COMPORTAMENTALES COMPETENCIALES}

4.1 Comunicación con 4.1.1 Mantiene relaciones adecuadas y de respeto con sus superiores. los jefes. 4.1.2 Escucha y analiza activamente ideas y opiniones diferentes.

4.2 Comunicación con 4.2.1 Mantiene relaciones adecuadas, de respeto y cooperación con sus los colegas. colegas de trabajo

4.2.2 Propone ideas con coherencia y claridad orientado a que exista una mayor cohesión entre el equipo de trabajo.

4.2.3 Escucha y analiza activamente ideas y opiniones diferentes de sus colegas.

4.3 Comunicación con 4.3.1 Fomenta un diálogo cálido, respetuoso y cordial con los clientes. los clientes. 4.3.2 Ante situaciones problemáticas o de conflictos mantiene la compostura, procurando visualizar desde la otra posición las posibles soluciones a negociar.

4.3.3 Escucha y analiza activamente ideas y opiniones diferentes.

4.3.4 Sabe manejar la incertidumbre ante el desconocimiento o la duda del cliente a la hora de realizar su pedido.

4.3.5 Trasmite las características deseadas de la imagen corporativa y los valores de la empresa.

\begin{tabular}{c}
$\begin{array}{c}\text { Nombre de la } \\
\text { competencia }\end{array}$ \\
\hline
\end{tabular}

Nivel de desarrollo óptimo de la competencia para el cargo: Avanzado

Definición de la competencia: Es la disposición para realizar el trabajo con base en el conocimiento de las necesidades y expectativas de los clientes externos e internos, reflejando un trato amable y cordial, interés por la persona y por la solución a sus problemas

\section{DIMENSIONES DESCRIPTIVOS COMPORTAMENTALES} COMPETENCIALES

\begin{tabular}{ll}
\hline 5.1 Escucha activa. & 5.1.1Tramita cualquier queja o sugerencia dada por los clientes \\
\hline 5.2 Respeto al cliente. & $\begin{array}{l}\text { 5.2.1 Atiende correctamente a los clientes aplicando las reglas de } \\
\text { cortesía. } \\
\end{array}$ \\
& 5.2.2 Muestra un trato cordial y amable. \\
\hline 5.3 Paciencia. & $\begin{array}{l}\text { 5.3.1 Se comporta de manera controlada, incluso ante clientes difíciles. } \\
\text { 5.3.2 Sabe autocontrolar sus emociones ante una disputa de un cliente, } \\
\text { superior o colega. }\end{array}$ \\
\hline 5.4 Tolerancia ante & $\begin{array}{l}\text { 5.4.1 Es capaz de afrontar situaciones conflictivas que se puedan } \\
\text { presentar durante el servicio gastronómico. } \\
\text { situaciones críticas. }\end{array}$ \\
& $\begin{array}{l}\text { 5.4.2 Es capaz de ofrecer respuestas convincentes ante críticas, } \\
\text { insatisfacciones o reclamos de clientes. }\end{array}$ \\
\hline 5.5Nociones culturales & 5.5.1 Se mantiene actualizado sobre la vida cultural del país. \\
de la ciudad. & \\
\hline
\end{tabular}


5.5.2 Brinda información atractiva para los clientes relacionada con elementos culturales y económicos de la cuidad, como museos, opciones recreativas, cambio de moneda, transporte, etc.

\section{Propuesta batería de técnicas psicodiagnósticas para el proceso de selección en el cargo.}

Considerando que el proceso de selección se basa en las competencias del cargo, se tuvo en cuenta la triangulación de técnicas para hacerlo más confiable. Las técnicas propuestas permiten evaluar varias competencias (o indicadores de estas) $y$, a la vez, todas las competencias son evaluadas por, al menos, dos técnicas.

Tabla 7. Técnicas psicodiagnósticas versus competencias que evalúan

\begin{tabular}{|c|c|c|c|c|c|}
\hline Técnicas & $\begin{array}{l}\text { Trabajo } \\
\text { en } \\
\text { equipo }\end{array}$ & $\begin{array}{l}\text { Dominio } \\
\text { técnico de la } \\
\text { gastronomía }\end{array}$ & $\begin{array}{l}\text { Comportamiento } \\
\text { ético }\end{array}$ & $\begin{array}{l}\text { Comunicación } \\
\text { interpersonal }\end{array}$ & $\begin{array}{l}\text { Orientación } \\
\text { al servicio }\end{array}$ \\
\hline $\begin{array}{l}\text { Entrevista } \\
\text { semiestructurada }\end{array}$ & & $X$ & $X$ & $X$ & $X$ \\
\hline $\begin{array}{l}\text { Técnica } \\
\text { simulación } \\
\text { “Sabrías } \\
\text { manejarlo...?” }\end{array}$ & $X$ & $X$ & $X$ & $X$ & \\
\hline $\begin{array}{l}\text { Locus de control } \\
\text { del Rotter. }\end{array}$ & $X$ & $X$ & $X$ & $X$ & \\
\hline $\begin{array}{l}\text { Capacidad de } \\
\text { Trabajo en Equipo } \\
\text { y Comunicación } \\
\text { (CTEC). }\end{array}$ & $X$ & & $X$ & $X$ & $X$ \\
\hline
\end{tabular}

\section{Entrevista semiestructurada}

La entrevista constituye uno de los factores con más influencia en el proceso de selección de un candidato. Para que se traduzca en una verdadera ventaja para la organización debe orientarse a la exploración de objetivos específicos y de interés. Se recomienda, además, prestar atención a elementos como la higiene personal, el vocabulario, e indagar en la orientación a valores y el comportamiento ético del entrevistado. Dada la marcada influencia de la subjetividad del entrevistador, se recomienda orientar el espacio hacia la exploración de competencias relacionadas con el dominio de la especialidad y la orientación al servicio.

Principales aspectos para indagar:

$\checkmark$ Experiencia en la ocupación.

$\checkmark$ Lugar de preparación y formación. 
$\checkmark$ Cursos de superación terminados.

$\checkmark$ Motivos para abandonar el trabajo anterior.

$\checkmark$ Frecuencia de fluctuación.

Técnicas de simulación

Con la utilización de este tipo de técnicas se pretende ubicar al candidato en situaciones reales de toma de decisión típicas del cargo. De esta forma, demostrará sus habilidades de comunicación, buen vocabulario, buenos modales, carácter flexible y capacidad para gestionar situaciones conflictivas

El objetivo que se persigue es la evaluación de las competencias que se listan a continuación:

- Trabajo en equipo.

- Comunicación Interpersonal.

- Comportamiento ético.

Técnica de simulación “Sabrías manejarlo...?”

Esta técnica permite a la empresa conocer las fortalezas y debilidades del candidato. Además, es beneficiosa pues se puede diseñar teniendo en cuenta tanto las competencias laborales actuales como las futuras (en caso de que se modifiquen o perfeccionen).

1-La consigna de la situación 1 es la siguiente: "Usted llega al trabajo y un administrativo de la instalación le pide reunirse en su despacho con un tono de voz molesto. La reunión es para ver si usted sabe algo sobre un error detectado en el inventario del bar (o restaurante) del día anterior que debía realizar su compañero. (Usted sabe que el error cometido por el otro trabajador se debe a que él lo ayudó a terminar su trabajo, ya que no le alcanzaba el tiempo para hacerlo usted mismo). ¿Cómo solucionaría esta situación? Luego, se le pide al sujeto que reelabore el informe y que estructure parte de los resultados a partir de los gráficos o tablas requeridas.

2- La consigan de la situación 2 es la siguiente: "Usted se percata de que la cuenta de un cliente tiene errores, se le pide más de lo que realmente debe pagar. Por lo tanto, tiene la posibilidad de obviar el error y cobrarle de más” ¿Qué haría usted?

3-La consigna de la situación 3 es la siguiente: "Los clientes de una de las mesas de su área, al terminar el servicio, le dan una elevada propina. Usted debe decidir si compartirla toda con sus compañeros, o guardar la mayor parte para usted y compartir el resto”. 
Técnica: Capacidad de Trabajo en Equipo y Comunicación (CTEC).

Esta técnica es diseñada por los investigadores, inspirados en la técnica NEGO. En este caso, será utilizada para medir la capacidad de trabajo en equipo y comunicación interpersonal en la realización de la actividad laboral. De ahí que se proponga otro nombre. La técnica consiste en un conjunto de situaciones propias del cargo que deben ser completadas por la persona evaluada. Se mide desde el posicionamiento en la situación hasta la originalidad de los mensajes utilizados. Las situaciones diseñadas serán presentadas a continuación:
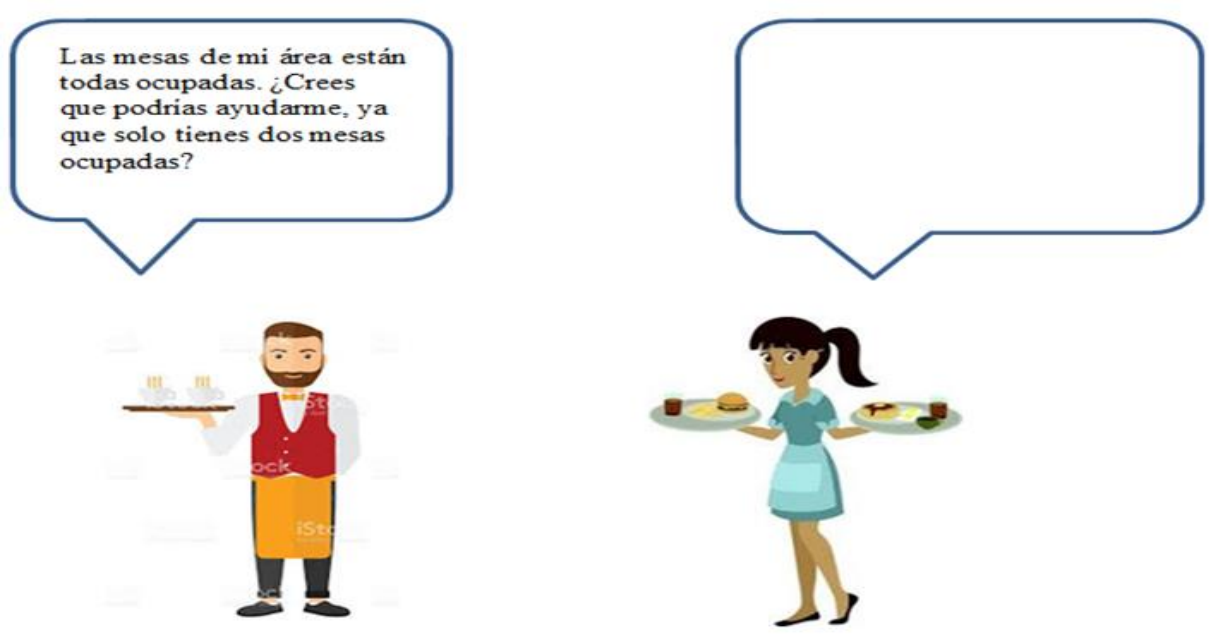

Figura 1. Situación 1

\section{Situación 2:}

Entra un cliente que, evidentemente, está teniendo un mal día. Usted lo atiende, pero al llevarle su pedido, sucede lo que a continuación se describe:

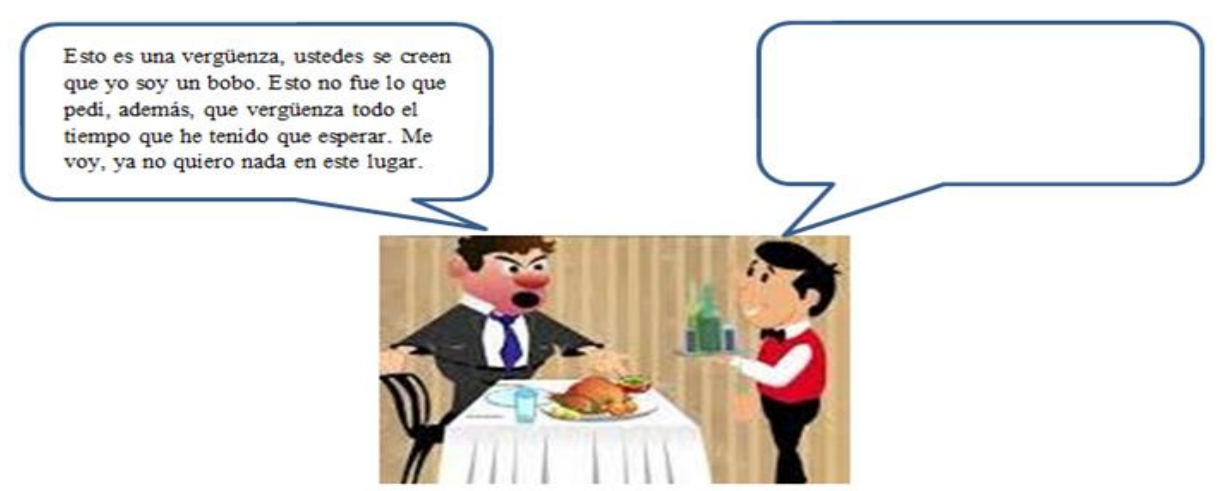

Figura 2. Situación 2 
Wimblu, Rev. Estud. de Psicología UCR, 16(1) 2021 (Enero-Junio): 75-101 /ISSN: 1659-2107

Se han utilizado situaciones reportadas como habituales por los expertos, pero pueden ser enriquecidas con otras experiencias en el desempeño del propio cargo.

De esta forma, queda demostrado cómo el perfil de competencias del cargo dependiente gastronómico puede ser utilizado en uno de los procesos de gestión de capital humano: la selección. La empresa puede disponer de este perfil para el diseño de otros procesos, entre ellos la evaluación, compensación, organización del trabajo y la capacitación.

\section{Conclusiones}

El perfil diseñado para el desempeño del cargo "dependiente gastronómico" de la empresa comprende las siguientes competencias claves: Trabajo en equipo, Dominio técnico de la gastronomía, Comportamiento ético, Comunicación interpersonal y Orientación al servicio; en ese orden de jerarquía. Estas deben ser consideradas en los procesos de gestión de capital humano para el cargo en cuestión.

Las condiciones laborales son un elemento de relevancia para el desempeño superior en el cargo. Con relación a ellas se diagnosticó que, en su mayoría, generan efectos funcionales para el desempeño de la actividad laboral. Sin embargo, el horario laboral extenso y el régimen de trabajo y descanso (trabajo en días alternos) generan efectos disfuncionales a mediano o largo plazo, como la fatiga y el estrés; por lo que es necesaria su modificación con vistas a prevenir la aparición de estados que afecten la salud de los dependientes y su desempeño laboral.

A partir de las competencias definidas para el perfil diseñado, se propone a la empresa una batería de técnicas para la selección de personal en el cargo “dependiente gastronómico”, que cumple con el criterio de la triangulación para otorgar mayor confianza en la evaluación. Dichas técnicas están conformadas por cuatro estrategias que evalúan los indicadores de las competencias claves. Se recomienda el entrenamiento de los evaluadores en su uso antes de la implementación. 


\section{Referencias bibliográficas}

Alles, Martha. "Perfil del puesto por competencias. Sepa cómo construirlo y evitar bajos desempeños posteriores". (2020). http://www.tblgroup.com.

Avila, Adalberto, y Medina, Arianne. "La Psicología y su relación con la efectividad de las organizaciones laborales". Tabloide de Universidad para Todos (2015). ISBN 978959-270-333-9.

Cáceres, Ana Beatriz. "Impacto de las condiciones laborales en los resultados del trabajo en cargos de organizaciones laborales de La Habana receptoras de prácticas de Psicología Laboral”. Tesis en opción al título de Licenciado en Psicología. Facultad de Psicología. Universidad de La Habana. 2020.

Cárdenas, Elia Teresa., Pérez, Olga Liset, González, Aliesky y Marrero, Fernando. “Manual de trabajo para diseñar los perfiles de cargos por competencias en las Universidades". Uniandes EPISTEME. Revista digital de Ciencia, Tecnología e Innovación 7, no. 2 (2020): 207-219. ISSN 1390-9150.

Cuesta, Armando. "Modelo Integrado de gestión humana y del conocimiento: una tecnología de aplicación”. Revista venezolana de Gerencia 17, no. 57 (2012): 86-98.

Díaz, Yohan, Cruz, Miguel, Pérez, Milagro de la Caridad y Ortiz, Tania. "El método criterio de expertos en las investigaciones educacionales: visión desde una muestra de tesis doctorales". Revista Cubana de Educación Superior 39, no.1, e18 (2019). ISSN 0257 4314.

Hernández, R., Fernández, C., \& Baptista, P. Metodología de la Investigación. México: Mc. Grau Hill Education. (2014).

Labrada, Edel. "Perfil de competencias del personal de servicios gastronómicos en la UEB servicios a trabajadores de la empresa de construcción y montaje de Las Tunas". Tesis en opción al título académico de Máster en Dirección. Facultad de Ciencias Económicas Centro de Estudios de Dirección. Universidad de Las Tunas. 2018.

Medina, Arianne; Martínez, Marta; Vitier, Amanda; Blanco, Yaima y Hernández, Midalys. "Manual de Trabajo Extraclase de Psicología Laboral en las Organizaciones para el 
Wimblu, Rev. Estud. de Psicología UCR, 16(1) 2021 (Enero-Junio): 75-101 /ISSN: 1659-2107

diseño de perfiles de cargos por competencias”. Facultad de Psicología. Universidad de La Habana. 2019.

Medina, Arianne y Vitier, Amanda. "El perfil de competencias: comparación de procedimientos para su diseño en el ámbito académico y empresarial cubano". Integración Académica en Psicología 5, no. 15 (2016b): 107-124. ISSN 2007-5588.

Medina Macías, Arianne y Avila Vidal, Adalberto. “¿Gestión de riesgos psicosociales? Un S.O.S. en organizaciones cubanas". Psicología: Teoría e Práctica 21, no.2 (2019):191-210.

Medina, Arianne y Vitier, Amanda. “La gestión de competencias influye en la efectividad organizacional? Apuntes para la reflexión desde la práctica en empresas cubanas”. Revista Wimb lu 11, no.2 (2016a): 59-85.

Medina, Arianne y Vitier, Amanda. "Socializando buenas prácticas sobre diseño de perfiles de competencias”. Alternativas Cubanas en Psicología (ACUPSI) 4, no. 10 (2016): $57-82$.

Oficina Nacional de Normalización. Norma Cubana 3000:2007. Sistema de Gestión Integrada de Capital Humano-Vocabulario. Vedado. La Habana. Cuba. 2007.

Soltura, Ariel y Cuesta, Armando. "Diseño estratégico de perfiles de cargos por competencias. Una contribución al alineamiento del desempeño individual con el desempeño organizacional”. Ingeniería Industrial XXIX, no. 1 (2008): 52-56. http://www.redalyc.org/articulo.oa?id=360433565003. 


\section{Anexos}

\section{Anexo 1: Diario de actividades}

Dado que todos los expertos realizan las mismas actividades, solo con la diferenciación del área donde se desempeñan, la recogida se realizó de la siguiente forma:

Bar:

\begin{tabular}{ll}
\hline Horario & Actividad \\
\hline 10-11AM & Llegada y preparación del salón \\
\hline 11-11:30AM & Arreglarse y vestirse con la ropa adecuada \\
\hline 11:30-12PM & Almuerzo \\
\hline 12PM & Abrir y comenzar el servicio \\
\hline 5:30-6PM & Turnarse para comer \\
\hline 9:30-10PM & Turnarse para merendar \\
\hline 12AM & Cierran \\
\hline 12-1:30AM & Recogen el salón y lo dejan montado para el día siguiente \\
\hline
\end{tabular}

Restaurante

\begin{tabular}{ll}
\hline Horario & Actividad \\
\hline 7-7:30AM & Llegada y preparación del salón \\
\hline 7:30-8AM & Arreglarse y vestirse con la ropa adecuada \\
\hline $\mathbf{8 A M}$ & Abrir y comenzar el servicio \\
\hline $\mathbf{1 1 : 3 0 - 1 2 P M}$ & Almuerzo \\
\hline $\mathbf{5 : 3 0 - 6 P M}$ & Turnarse para comer \\
\hline $\mathbf{9 : 3 0 - 1 0 P M}$ & Turnarse para merendar \\
\hline $\mathbf{1 2 A M}$ & Cierran \\
\hline $\mathbf{1 2 - 1 : 3 0 A M}$ & Recogen el salón y lo dejan montado para el día siguiente \\
\hline
\end{tabular}

\section{Anexo 2. Orientaciones para la entrevista semiestructurada de condiciones laborales}

Consigna: El propósito de esta entrevista es conocer qué condiciones externas e internas se requieren para hacer bien el trabajo. Necesitamos que nos comente cuáles son las condiciones laborales relevantes para desempeñarse con éxito en su puesto y qué influencia 
tienen (tal como aparecen en su puesto de trabajo) en sus resultados de trabajo y en su estado de ánimo.

Sus opiniones nos ayudarán a definir cómo su organización puede contribuir a que usted tenga un mejor desempeño en su puesto de trabajo a partir de la valoración de sus condiciones laborales.

\section{Datos Generales:}

Cargo:

Años de experiencia en el cargo:

Años de experiencia en la organización

Área o departamento al que pertenece el cargo:

Misión del cargo:

Nota: La entrevista debe recorrer todas las condiciones laborales mencionadas en el modelo de análisis y otras que no aparezcan y se consideren relevantes (se recomienda usar el modelo como hoja de anotación en caso de no aplicarse como encuesta). Se requiere una preparación previa de la misma, incluyendo a los entrevistadores que deben dominar en profundidad todo su contenido. En la información que se recoja debe quedar claro cuándo una condición es relevante para el desempeño superior en el cargo (o sea, sin ella es imposible desempeñarse de manera exitosa) y, además, si esa condición tiene una influencia beneficiosa o perjudicial para los resultados de trabajo de la forma en que se manifiesta hoy en día.

Se recomienda comenzar por las condiciones laborales más fáciles de comentar para los expertos, como las organizativas: horario laboral, organización de turnos de trabajo (fijos o rotativos), régimen de rotación (si son turnos rotativos), sistema de pausas en una jornada laboral, tipo de pago (a tiempo o por rendimiento), etc.

Ojo: las condiciones laborales deben quedar descritas; por ejemplo: horario laboral de 8 horas diarias durante 5 días a la semana (de lunes a viernes), desde las 8:00 am a 5:00 pm. o horario laboral abierto o flexible.

Deben incluir las preguntas cuando se refieran a los riesgos acerca de los accidentes.

¿Ha sufrido durante su experiencia en el cargo algún accidente o incidente laboral? Coméntenos sobre ellos, y diga cuáles fueron sus causas. 


\section{Anexo 3. Guía de entrevista de Incidentes Críticos}

A continuación, te ofrecemos un ejemplo de guía de entrevista de incidentes críticos para aplicar a cada uno de los expertos. Según las particularidades del cargo objeto de estudio, las características de la organización, así como de las respuestas de los expertos, podrá ser modificada, o mantenida, eso dependerá de su valoración:

Experiencia positiva, de éxito:

1. ¿Podría relatar una experiencia positiva, de éxito que haya vivido durante su trabajo como "x" (cargo que se está estudiando) en esta organización?

2. ¿Qué competencias/cualidades considera que haya puesto en práctica para lograr el éxito en dicha experiencia?

3. ¿Recibió ayuda para llevar a cabo con éxito la tarea que se propuso? ¿De quién, específicamente?

4. ¿Cuál fue la respuesta de su jefe inmediato ante el éxito de esa experiencia?

Experiencia negativa, de fracaso:

1. ¿Podría relatar una experiencia negativa, de sentimiento de fracaso que haya vivido durante su trabajo como " $\mathrm{x}$ " (cargo que se está estudiando) en esta organización?

2. ¿Qué competencias/cualidades considera que estuvieron ausentes, por lo que se derivó en un fracaso?

3. ¿Qué factores o personas intervinieron en dicha experiencia?

4. ¿Recibiste ayuda para revertir la situación? ¿De quién específicamente?

5. ¿Cuál fue la respuesta de su jefe inmediato ante el fracaso de esta actividad?

A partir de esas experiencias que usted nos ha comentado ¿Cuáles son los criterios de éxito en el desempeño del cargo " $\mathrm{x}$ "?

\footnotetext{
(c) (i) Esta obra está bajo una licencia de Creative Commons ReconocimientoEY No sa NoComercial-CompartirIgual 4.0 Internacional
} 